\title{
Diagnostic value of puncture feeling combined with BRAF V600E mutation in repeat US-FNA biopsy of Bethesda III thyroid nodules
}

\author{
$\mathrm{Li} \mathrm{Li}^{1,2}$, Peipei $\mathrm{Li}^{2}$, Xiao Chen ${ }^{2}$, Lin Kang ${ }^{3}$, Yuquan $\mathrm{Ye}^{1,2} \wedge$ \\ ${ }^{1}$ School of Graduate, Hebei Medical University, Shijiazhuang, China; ${ }^{2}$ Department of Ultrasound, Hebei General Hospital, Shijiazhuang, China; \\ ${ }^{3}$ Department of Pathology, Hebei General Hospital, Shijiazhuang, China \\ Contributions: (I) Conception and design: Y Ye, L Li; (II) Administrative support: None; (III) Provision of study materials or patients: None; (IV) \\ Collection and assembly of data: L Kang; (V) Data analysis and interpretation: X Chen, P Li; (VI) Manuscript writing: All authors; (VII) Final \\ approval of manuscript: All authors. \\ Correspondence to: Yuquan Ye, MD. NO. 348 of West He-Ping Road, Xin-Hua District, Shijiazhuang 050051, China. Email: hbghyyq@163.com.
}

\begin{abstract}
Background: The diagnosis and treatment of Bethesda III thyroid nodules has always been controversial. Our aim is to study the value of puncture feeling combined with BRAF V600E mutation detection in the diagnosis of Bethesda III thyroid nodules in repeat fine needle aspiration (FNA).

Methods: From January 1, 2017 to December 31, 2021, a total of 1,114 thyroid nodules were included, of which Bethesda III thyroid nodules accounted for $12.1 \%$. We analyzed the correlation between puncture feeling and postoperative pathology. Then, the diagnostic value of puncture feeling in Bethesda III thyroid nodules and the mutation rate of BRAF V600E in repeated FNA and its correlation with puncture feeling were analyzed.
\end{abstract}

Results: The results showed that there was a significant correlation between puncture feeling and postoperative pathology in the 1114 thyroid nodules $(\mathrm{P}<0.001)$. Additionally, 93.4\% (299/320) of the thyroid nodules with soft puncture feeling were benign, $89.0 \%(460 / 517)$ of the thyroid nodules with hard puncture feeling were malignant, and 93.1\% (258/277) of the thyroid nodules with a puncture feeling of hard with grittiness were malignant. There was high consistency of puncture feelings among different operators $(\mathrm{P}<0.001)$. The distribution of puncture feeling between benign and malignant thyroid nodules was significantly different $(\mathrm{P}<0.001)$ in Bethesda III thyroid nodules. In the puncture feeling ROC curve of Bethesda III thyroid nodules based on pathology, the AUC was 0.895 (95\% CI: 0.817-0.973, P<0.001). In repeat FNA, BRAF V600E was significantly correlated with puncture feeling $(\mathrm{P}<0.001)$. BRAF V600E mutation was found in $22(68.8 \%)$ malignant nodules.

Conclusions: Puncture feelings of hard and hard with grittiness were malignant risk factors. Repeated FNA and BRAF V600 mutation detection should be performed for Bethesda III thyroid nodules with a hard or hard with grittiness puncture feeling.

Keywords: Bethesda III thyroid nodules; puncture feeling; benign; malignant; BRAF V600E

Submitted Apr 02, 2021. Accepted for publication Jun 02, 2021.

doi: 10.21037 /gs-21-302

View this article at: https://dx.doi.org/10.21037/gs-21-302

\section{Introduction}

Thyroid nodules are a widespread medical problem, with a prevalence rate of $19 \%$ to $68 \%$ in the general population
$(1,2)$. Thyroid cancer is one of the fastest-growing endocrine cancers in recent years. Its etiology is complex, and some of its mechanisms may be related to elevated TSH. TSH is

\footnotetext{
$\wedge$ ORCID: 0000-0002-1335-7477.
} 
a thyroid stimulating hormone. TSH regulates the growth of thyroid follicular cells through a signal transduction pathway mediated by adenylate cyclase (CAMP). Excessive TSH levels are related to the occurrence of thyroid cancer. Specific reasons include iodine deficiency and high-iodine diet, radiation to destroy the thyroid, sex hormones, genetic factors, and other thyroid diseases, nodular goiter, and congenital hyperplastic goiter without adequate treatment for a long time. At present, ultrasound-guided fine needle aspiration (US-FNA) biopsy has been the most accurate method for the diagnosis of thyroid nodules (3). The Bethesda system for reporting thyroid cytopathology has been extensively used worldwide, with each category documenting the risk of malignancy, and providing management advice and recommending repeat FNA for Bethesda III thyroid nodules $(4,5)$. In the Bethesda system, about $15-30 \%$ of thyroid nodules are classified as Bethesda III category after FNA $(4,6)$, that is, indeterminate atypia of undetermined significance (AUS) and follicular lesion of undetermined significance (FLUS). The inability to distinguish benign from malignant thyroid nodules is the main limitation of USFNA for Bethesda III thyroid nodules (3). The probability of malignancy of Bethesda III thyroid nodules is estimated to be $10-30 \%$ (7), and the malignant incidence of Bethesda III thyroid nodules is difficult to determine due to only a small portion of these cases being surgically removed. Those who have undergone removal only represent some patients whose clinical, ultrasound, or molecular findings are at risk of malignancy (8).

The treatment of Bethesda III thyroid nodules is different in clinical practice. According to the ultrasonic manifestations, clinical risk factors, and patients' wishes, the diagnosis and treatment methods include repeat FNA, regular follow-up, gene detection, or diagnostic surgical treatment, amongst others. It is reported that the diagnostic accuracy of Bethesda III thyroid nodules after repeat FNA is $65 \%$ (3). Among the molecular markers, BRAF V600E mutation is the most common mutation in papillary thyroid carcinoma (PTC) (9), accounting for $28-83 \%$ of all gene mutations and $90 \%$ of all BRAF mutations (10-12). However, BRAF V600E mutations were not detected in normal thyroid tissues or benign lesions (13-16). Therefore, when the cell pathological diagnosis of the initial FNA is Bethesda III category, the thyroid nodule is too small or the cell mass is insufficient for diagnosis, repeated FNA combined with BRAF V600E can help confirm the diagnosis of malignant thyroid nodules.

Because only a small portion of Bethesda III thyroid nodules may be malignant, and to avoid excessive diagnosis and treatment, which patients need repeat FNA and which patients can be followed up by ultrasonography should be determined. Moreover, patients with repeat FNA should be combined with BRAF V600E mutation detection, which can avoid missed diagnosis of malignant thyroid nodules, and can also reduce benign thyroid nodules that require unnecessary surgery for diagnosis, and reduce the economic burden of patients. During the process of FNA, the operator can feel the texture when the needle tip enters into the thyroid nodule, which is the puncture feeling, and the texture of different thyroid nodules can be completely different. One can get various feelings in the process of FNA, such as needle getting stuck, hard to pull, grittiness and so on (17). FNA of thyroid nodules is not the most perfect diagnostic method, there are false positive rates and false negative rates. Hence, the puncture feeling may provide evidence for the diagnosis of thyroid nodules. It has been proven that puncture feeling is of great value in the diagnosis of thyroid nodules, and puncture feeling of grittiness is one of the risk factors of malignant thyroid nodules (17). However, some malignant thyroid nodules do not have calcification, and the puncture feeling is hard and not accompanied by grittiness. Therefore, the puncture feeling in this study was divided into 3 types: soft, hard, and hard with grittiness. Thus far, there is no study on the diagnostic value of puncture feeling in Bethesda III thyroid nodules. To reduce the occurrence of such uncertain results, the purpose of this study was to explore the diagnostic value of puncture feeling in Bethesda III thyroid nodules and the correlation between BRAF V600 mutation and postoperative pathology and puncture feeling during repeat FNA.

We present the following article in accordance with the STARD reporting checklist (available at https://dx.doi. org/10.21037/gs-21-302).

\section{Methods}

\section{Patients}

All procedures performed in this study involving human participants were in accordance with the Declaration of Helsinki (as revised in 2013). The study was approved by institutional ethics board of Hebei General Hospital (NO.12). From January 1, 2017 to December 31, 2020, a total of 1,114 thyroid nodules were included. A process diagram of patient screening is shown in Figure 1. The inclusion criteria included preoperative FNA, puncture 


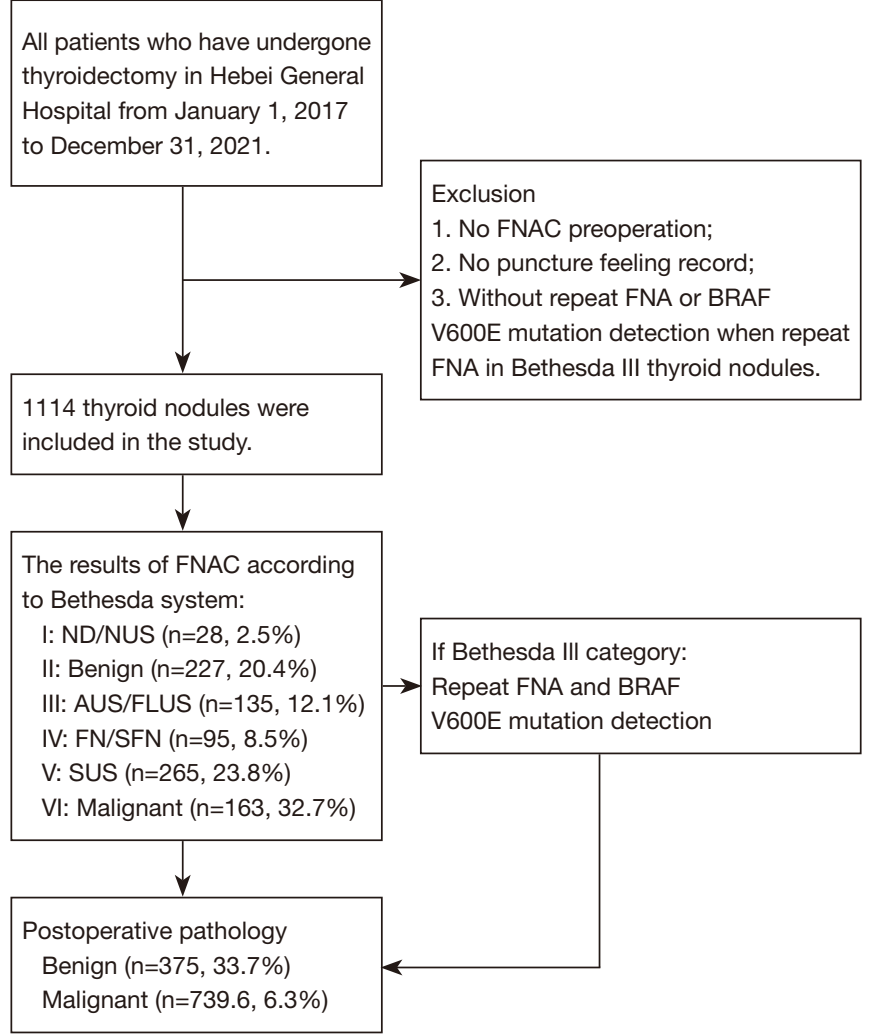

Figure 1 Process diagram of patient screening. FNAC, fine needle aspiration cytology.

feeling records, and BRAF V600E mutation testing in Bethesda III thyroid nodules during repeat FNA. Patients who did not meet the above criteria were excluded from the study. Prior to FNA, all patients signed informed consent for special treatment. Repeat FNA was recommended for Bethesda III thyroid nodules after 3 months, and the operator informed the patients of the significance and related cost of the BRAF V600E test before repeat FNA, all of which were tested with the consent of the patient. Repeat FNA was performed by another experienced operator.

\section{The operation process of US-FNA}

The operator used the Philips EPIQ 7 color Doppler ultrasound instrument made by Philips in the United States, with an L12-5 linear array probe and a frequency of 5-12 $\mathrm{MHz}$. The patient was placed in the supine position before the operation, the target nodule was scanned and located by ultrasound, and the puncture path of FNA was chosen. The puncture needle was a $23 \mathrm{G}$ or $25 \mathrm{G}$ puncture needle from Japanese Hakko Co., Ltd. The surgeon used iodophor for disinfection, aseptic hole towels were spread, and 1 $\mathrm{ml} 2 \%$ lidocaine was used for local infiltration anesthesia. Under the guidance of ultrasound, the operator pierced the puncture needle into the nodule along the puncture path, with rapid sampling and rapid smear fixation. The assistant sent the specimens to the pathology department for cytological diagnosis by a professional pathologist.

\section{Classification criteria}

The diagnostic results of FNA were classified according to the Bethesda system (7). Puncture feeling referred to the texture of the nodules felt by the operator when the needle entered into the nodule, including nonresistance, stickiness, resistance, and grittiness. In this study, puncture feeling was divided into the following 3 categories: soft, hard, and hard with grittiness. Soft: the texture of thyroid nodules was similar to or softer than that of the normal thyroid parenchyma, there was no obvious sense of resistance when the fine needle entered the nodule, and the gland did not shift during the process of FNA. Hard: the texture of the thyroid nodule was hard, and there was an obvious sense of resistance or stickiness when the puncture needle entered the nodule, and after the puncture needle entered into the nodule, the gland had any degree of displacement with the lifting and insertion of the needle. Hard with grittiness: the thyroid nodule was hard, and when the puncture needle entered into the nodule there was an obvious sense of resistance, accompanied by a sense of grittiness.

\section{Statistical analysis}

SPSS19.0 statistical software was used for statistical analysis. The chi-square test and Fisher's exact test were used to compare the categorical variables. According to the postoperative pathological results, the receiver operating characteristic (ROC) curve was constructed and the area under the curve (AUC) was calculated. $\mathrm{P}<0.05$ was statistically significant.

\section{Results}

\section{Clinicopathological characteristics of the patient}

A total of 1,114 thyroid nodules were included in this study. The clinicopathological characteristics of the patients are shown in Table 1 . The average age was $44.5 \pm 12.8$ years old. 
Table 1 Clinicopathological characteristics of all the patients

\begin{tabular}{lc}
\hline Characteristics & No. (\%) \\
\hline Age (years), mean \pm SD & $44.5 \pm 12.8$ \\
Gender & $323(29.0)$ \\
Male & $791(71.0)$ \\
Female & \\
FNA Bethesda category & $28(2.5)$ \\
I & $227(20.4)$ \\
II & $135(12.1)$ \\
III & $95(8.5)$ \\
IV & $265(23.8)$ \\
V & $364(32.7)$ \\
VI & \\
Puncture feeling & $320(28.7)$ \\
Soft & $517(46.4)$ \\
Hard & $277(24.9)$ \\
Hard with grittiness & $1,114(100.0)$ \\
Total & \\
\hline
\end{tabular}

FNA, fine needle aspiration.

The number of female patients was 2.4 times higher than that of males (791, $71.0 \%$ vs. 323, 29.0\%). According to the Bethesda system, there were 28 (2.5\%), 227 (20.4\%), $135(12.1 \%), 95(8.5 \%), 265(23.8 \%)$, and $364(32.7 \%)$ thyroid nodules in categories I-VI, respectively. Puncture feeling was recorded for all thyroid nodules, of which $320(28.7 \%)$ were soft, $517(46.4 \%)$ were hard, and 277 (24.9\%) were hard with grittiness. Firstly, we analyzed the correlation between puncture feeling and postoperative pathology. As shown in Table 2, there was a significant correlation between puncture feeling and postoperative pathology, and the difference was statistically significant $(\mathrm{P}<0.001)$. Of the 1114 thyroid nodules, 739 (66.3\%) were malignant and 375 (33.7\%) were benign. Among them, 93.4\% (299/320) of the thyroid nodules with soft puncture feeling were benign, $89.0 \%$ (460/517) of the thyroid nodules with hard puncture feeling were malignant, and as high as 93.1\% (258/277) of the thyroid nodules with puncture feeling of hard with grittiness were malignant. The results show that thyroid nodules with soft puncture feeling are more likely to be benign. Furthermore, the puncture feelings of hard and hard with grittiness suggest that these thyroid nodules are at risk of malignancy, especially hard with grittiness nodules which are the most likely to be malignant.

\section{Analysis of the consistency of puncture feeling of different operators}

In this study, $12.1 \%(135$ to 1,114$)$ of thyroid nodules were classified as Bethesda III after FNA, and the diagnosis and treatment of such nodules were controversial. The above results show that there is a correlation between puncture feeling and postoperative pathology, so does this have guiding significance for Bethesda III thyroid nodules? We further analyzed the consistency of puncture feeling among different operators. During repeat FNA of Bethesda III thyroid nodules, the operation was performed by another operator, and the puncture feeling was recorded. The results in Table 3 showed that there was a high consistency of puncture feeling among different operators $(\mathrm{P}<0.001$, $\left.\chi^{2}=144.831, \kappa=0.746\right)$.

\section{The value of puncture feeling in the diagnosis of Bethesda III thyroid nodules}

We also analyzed the correlation between puncture feeling and postoperative pathology of Bethesda III thyroid nodules, and the results were also significant (Table 4, $\mathrm{P}<0.001)$. Of the Bethesda III nodules, $23.7 \%$ (32/135) were malignant and $76.3 \%$ (103/135) were benign. Furthermore, 95.0\% $(95 / 100)$ of the thyroid nodules with soft puncture feeling were benign, $66.7 \%(14 / 21)$ of the thyroid nodules with hard puncture feeling were malignant, and $92.9 \%(13 / 14)$ of the thyroid nodules with puncture feeling of hard with grittiness were malignant. According to the postoperative pathology, the ROC curve of Bethesda III nodules was obtained as shown in Figure 2, and the AUC was 0.895 (95\% CI: $0.817-0.973, \mathrm{P}<0.001$ ), which proved that the puncture feeling was of high value in the diagnosis of Bethesda III nodules. Hard and hard with grittiness were risk factors for malignancy, and soft indicated that the thyroid nodule was likely to be benign. However, $33.3 \%$ of the thyroid nodules with hard puncture feeling were benign, which could be distinguished from malignant nodules through ultrasound image features, avoiding excessive diagnosis and treatment.

\section{The significance of BRAF V600E mutation in repeated FNA in Bethesda III thyroid nodules}

All Bethesda III thyroid nodules in this study were detected 
Table 2 Correlation between puncture feeling and postoperative pathology in all the thyroid nodules

\begin{tabular}{|c|c|c|c|c|}
\hline Puncture feeling & Total, n (\%) & \multicolumn{2}{|c|}{ Postoperative pathology, n (\%) } & $P$ value \\
\hline Soft & $320(28.7)$ & $21(6.6)$ & $299(93.4)$ & $<0.001$ \\
\hline Hard & $517(46.4)$ & $460(89.0)$ & $57(11.0)$ & \\
\hline Hard with grittiness & 277 (24.9) & $258(93.1)$ & $19(6.9)$ & \\
\hline
\end{tabular}

Table 3 The consistency of puncture feeling between operator A and operator B

\begin{tabular}{lccccc}
\hline \multirow{2}{*}{ Initial FNA (operator B) } & \multicolumn{3}{c}{ Repeat FNA (operator A) } & \multirow{2}{*}{$\chi^{2}$} & \multirow{2}{*}{ P value } \\
\cline { 2 - 5 } & Soft & Hard & Hard with grittiness & 144.831 & $<0.001$ \\
\hline Soft & 93 & 7 & 0 & 0.746 \\
Hard & 2 & 16 & 3 & 11 \\
Hard with grittiness & 0 & 3 & & \\
\hline
\end{tabular}

FNA, fine needle aspiration.

Table 4 Correlation between puncture feeling and postoperative pathology in Bethesda III thyroid nodules

\begin{tabular}{lccc}
\hline \multirow{2}{*}{ Puncture feeling } & Total, $\mathrm{n}(\%)$ & \multicolumn{2}{c}{ Postoperative pathology, $\mathrm{n}(\%)$} \\
\cline { 3 - 4 } & & Malignant & Benign \\
\hline Soft & $100(74.0)$ & $14(6.0)$ & $95(95.0)$ \\
Hard & $21(15.6)$ & $13(92.9)$ & $7(33.3)$ \\
Hard with grittiness & $14(10.4)$ & $32(23.7)$ & $1(7.1)$ \\
Total & $135(100.0)$ & $103(76.3)$ \\
\hline
\end{tabular}

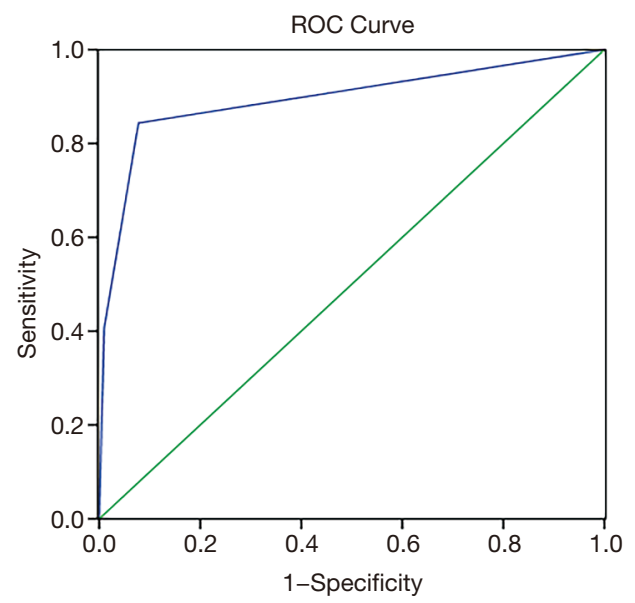

Figure 2 Puncture feeling ROC curve of Bethesda category III thyroid nodules based on pathology. The AUC was $0.895(95 \%$ CI: 0.817-0.973), $\mathrm{P}<0.001$. by repeat FNA and BRAF V600E mutation with the consent of the patients, and the correlation between BRAF V600E and postoperative pathology and puncture feeling was analyzed. As shown in Table 5, BRAF V600E was correlated with postoperative pathology and puncture feeling, both with $\mathrm{P}$ value $<0.001$. BRAF V600E mutation was found in $22(68.8 \%)$ malignant nodules, but no mutation was found in benign nodules. Furthermore, $85.7 \%$ (12/22) of the nodules with gene mutations had a puncture feeling of hard with grittiness, $38.1 \%$ of the nodules with gene mutations had hard puncture feeling, and only 2.0\% (2/100) of the nodules with soft puncture feeling had BRAF V600E mutations. The above results showed that the incidence rate of BRAF V600E mutation in malignant thyroid nodules in repeat FNA was $68.8 \%$. Among the nodules with gene mutations, the nodules with a puncture feeling of hard with 
Table 5 Distribution of BRAF V600E mutations in postoperative pathology and puncture feeling in Bethesda III thyroid nodules

\begin{tabular}{lcc}
\hline & BRAF V600E,$+ \mathrm{n}(\%)$ & BRAF V600E -, n (\%) \\
\hline Postoperative pathology & $22(68.8)$ & $10(31.3)$ \\
Malignant & $0(0.0)$ & $103(100.0)$ \\
Benign & & \\
Puncture feeling & $2(2.0)$ & $98(98.0)$ \\
Soft & $8(38.1)$ & $13(61.9)$ \\
Hard & $12(85.7)$ & $2(14.3)$ \\
Hard with grittiness & & $<0.001$ \\
\hline
\end{tabular}

grittiness had the most, and $38.1 \%$ of the nodules with hard puncture feeling had BRAF V600E mutations. However, there were few cases of Bethesda III thyroid nodules in this study, and a sufficient number of cases need to be examined in the future to confirm these results.

\section{Adverse events}

Only a small number of patients in this study had mild pain during puncture, and fewer patients had bleeding complications, but they could stop bleeding after compression.

\section{Discussion}

It is reported that thyroid nodules are usually found during physical examination, and the detection rate of thyroid nodules is about $19-68 \%$ (3). US-FNA is the most commonly used method for the diagnosis of thyroid nodules, but the diagnosis and treatment of Bethesda III thyroid nodules are problematic for endocrinologists and surgeons. This study found that puncture feeling plays an important role in guiding the further diagnosis of Bethesda III thyroid nodules.

The results of the correlation analysis between puncture feeling and postoperative pathology of the 1,114 nodules included in this study (Table 2) proved that there was a significant correlation between them $(\mathrm{P}<0.001)$. Puncture feelings of hard and hard with grittiness were malignant risk factors, while nodules with soft puncture feeling were more likely to be benign. This is because the puncture feeling is closely related to the pathological structure of thyroid nodules. The most common pathological type of malignant thyroid nodules is PTC. Its cytopathological features are as follows: epithelial cells are arranged in a papillary pattern, and the nucleolus, nuclear sulcus, and nuclear pseudoinclusion bodies can be seen. Compared with normal thyroid tissue, there are very few follicular glia. With the continuous development of the patient's condition, psammoma bodies can be found in most PTC lesions, which is caused by tissue degeneration and necrosis produced by insufficient cell blood supply, and then calcium salt is produced and deposited. It is precisely because of this that the operator will feel hard when the needle tip enters the nodule, and the puncture feeling of grittiness is caused by the psammoma bodies. The most common benign nodules are nodular goiter and follicular adenoma. Nodular goiter consists of dilated follicles in varying degrees, and the cavity is full of colloids. Follicular adenoma is composed of hyperplastic follicular epithelium, and there are a large number of follicles, most of which are edema-like loose fibrous stroma. Therefore, the needle tip can smoothly enter the interior of benign nodules during FNA, which is soft and has no obvious sense of resistance.

Of the 1,114 thyroid nodules, there were 135 (12.1\%) Bethesda III thyroid nodules, among which 32 (23.7\%) were pathologically proven to be malignant. The results of Table 3 in this study showed that puncture feeling had good repeatability among different operators and could be used as a reliable indicator for evaluating the nature of thyroid nodules. Kuru et al. demonstrated that the incidence of malignancy of thyroid nodules whose first FNA result was AUS/FLUS was significantly higher after repeat FNA than that of nonrepetitive FNA (18). Repeat FNA is recommended for those nodules whose first FNA result is AUS/FLUS. However, not all Bethesda III thyroid nodules require repeated FNA. The results in Table 4 of this study further confirmed that in Bethesda III thyroid nodules, there was a significant difference in the distribution of puncture feeling between benign and malignant nodules 
$(\mathrm{P}<0.001)$. We found that $92.9 \%$ of the thyroid nodules with puncture feeling of hard with grittiness were malignant, $66.7 \%$ of the hard thyroid nodules were malignant, and $95.0 \%$ of the soft thyroid nodules were benign. The results of Figure 2 also confirmed the diagnostic value of puncture feeling in Bethesda III thyroid nodules, with an AUC of 0.895 (95\% CI: $0.817-0.973, \mathrm{P}<0.001$ ). Therefore, if the puncture feeling of primary FNA in Bethesda III thyroid nodules is hard with grittiness, repeat FNA should be performed, as the risk of malignancy of such nodules is high. If the puncture feeling is hard, it should be analyzed together with ultrasound images, and if there is still a malignant risk, repeat FNA should be carried out to avoid a missed diagnosis. Some studies have shown that the stiffness of Hashimoto's thyroiditis and subacute thyroiditis will increase $(19,20)$, so when the puncture feeling of Bethesda III thyroid nodules is hard, it is sometimes impossible to distinguish inflammatory lesions from malignant nodules by puncture feeling alone. Thus, repeat FNA is needed to further confirm the diagnosis. In this study, when the puncture feeling of Bethesda III thyroid nodules was soft, 95.0\% were benign, ultrasound follow-up can be done.

Previous studies have shown that the most common molecular abnormality in thyroid cancer is the BRAF V600E mutation, which is a useful diagnostic marker (21-25). Other studies have shown that BRAFV600E analysis can improve the diagnostic accuracy of FNA and reduce the false negative rate, especially for AUS/FLUS (26). In this paper, the correlation analysis between BRAF V600E mutation and postoperative pathology and puncture feeling in repeat FNA of Bethesda III thyroid nodules in Table 5 showed that $68.8 \%$ of malignant nodules had mutations. Compared with the results of the Bethesda III thyroid nodules at the first FNA, BRAF V600E mutation has important guiding significance for the next step of diagnosis and treatment for clinicians. Moreover, most of the mutated nodules had the puncture feeling of hard with grittiness $(85.7 \%)$ and hard (38.1\%). However, the reason for the correlation between BRAF V600E mutation and puncture feeling was not clear. Therefore, when the first FNA results of thyroid nodules are in the Bethesda III category, if the puncture feeling is hard or hard with grittiness, and there are signs of malignancy on the ultrasound image, then the patient should be strongly recommended to perform repeated FNA combined with BRAF V600E mutation detection. The combined diagnosis is helpful for ensuring that patients with malignant nodules undergo surgical resection in time, and to avoid overtreatment in patients with benign nodules.

\section{Conclusions}

Puncture feeling is helpful to distinguish the nature of thyroid nodules on the basis of two-dimensional ultrasound. Moreover, puncture feeling has good repeatability among different operators, and can be used as a reliable indicator for evaluating the nature of thyroid nodules. When the puncture feeling is hard or hard with grittiness during the process of FNA, the thyroid nodule has a malignant tendency, while a soft puncture feeling indicates that the nodule is more likely to be benign. Therefore, when the initial FNA result is classified as Bethesda III category, if there is still a malignant risk in the combination of ultrasound images with puncture feeling of hard or hard with grittiness, repeat FNA and detection of BRAF V600E mutation should be carried out. This can provide a reliable basis for surgeons to devise treatment strategies, avoid excessive diagnosis and treatment, and reduce the economic burden of patients.

However, this study has some limitations. There was an insufficient number of Bethesda III thyroid nodules and thyroid nodules with BRAF V600E mutations. In the follow-up study, with the increase in the number of patients, we will conduct further analyses and research to increase the reliability of the study.

\section{Acknowledgments}

Funding: None.

\section{Footnote}

Reporting Checklist: The authors have completed the STARD reporting checklist. Available at https://dx.doi. org/10.21037/gs-21-302

Data Sharing Statement: Available at https://dx.doi. org/10.21037/gs-21-302

Conflicts of Interest: All authors have completed the ICMJE uniform disclosure form (available at https://dx.doi. org/10.21037/gs-21-302). The authors have no conflicts of interest to declare.

Data Availability: Our data can be shared publicly by the mailbox of the correspondent author. However, in order to prevent the disclosure of the original confidential data, our organization allows us to upload the original data only after the article is accepted. 
Ethical Statement: The authors are accountable for all aspects of the work in ensuring that questions related to the accuracy or integrity of any part of the work are appropriately investigated and resolved. All procedures performed in this study involving human participants were in accordance with the Declaration of Helsinki (as revised in 2013). The study was approved by institutional ethics board of Hebei General Hospital (NO.12). Prior to FNA, all patients signed informed consent for special treatment.

Open Access Statement: This is an Open Access article distributed in accordance with the Creative Commons Attribution-NonCommercial-NoDerivs 4.0 International License (CC BY-NC-ND 4.0), which permits the noncommercial replication and distribution of the article with the strict proviso that no changes or edits are made and the original work is properly cited (including links to both the formal publication through the relevant DOI and the license). See: https://creativecommons.org/licenses/by-nc-nd/4.0/.

\section{References}

1. Tan GH, Gharib H. Thyroid incidentalomas: management approaches to nonpalpable nodules discovered incidentally on thyroid imaging. Ann Intern Med 1997;126:226-31.

2. Guth S, Theune U, Aberle J, et al. Very high prevalence of thyroid nodules detected by high frequency $(13 \mathrm{MHz})$ ultrasound examination. Eur J Clin Invest 2009;39:699-706.

3. Haugen BR, Alexander EK, Bible KC, et al. 2015 American Thyroid Association Management Guidelines for Adult Patients with Thyroid Nodules and Differentiated Thyroid Cancer: The American Thyroid Association Guidelines Task Force on Thyroid Nodules and Differentiated Thyroid Cancer. Thyroid 2016;26:1-133.

4. Cibas ES, Ali SZ; NCI Thyroid FNA State of the Science Conference. The Bethesda System For Reporting Thyroid Cytopathology. Am J Clin Pathol 2009;132:658-65.

5. Alexander EK, Heering JP, Benson CB, et al. Assessment of nondiagnostic ultrasound-guided fine needle aspirations of thyroid nodules. J Clin Endocrinol Metab 2002;87:4924-7.

6. Bongiovanni M, Spitale A, Faquin WC, et al. The Bethesda System for Reporting Thyroid Cytopathology: a meta-analysis. Acta Cytol 2012;56:333-9.

7. Cibas ES, Ali SZ. The 2017 Bethesda System for Reporting Thyroid Cytopathology. Thyroid 2017;27:1341-6.

8. Iskandar ME, Bonomo G, Avadhani V, et al. Evidence for overestimation of the prevalence of malignancy in indeterminate thyroid nodules classified as Bethesda category III. Surgery 2015;157:510-7.

9. Xing M, Westra WH, Tufano RP, et al. BRAF mutation predicts a poorer clinical prognosis for papillary thyroid cancer. J Clin Endocrinol Metab 2005;90:6373-9.

10. Kim SW, Lee JI, Kim JW, et al. BRAFV600E mutation analysis in fine-needle aspiration cytology specimens for evaluation of thyroid nodule: a large series in a BRAFV600E-prevalent population. J Clin Endocrinol Metab 2010;95:3693-700.

11. Min HS, Lee C, Jung KC. Correlation of immunohistochemical markers and BRAF mutation status with histological variants of papillary thyroid carcinoma in the Korean population. J Korean Med Sci 2013;28:534-41.

12. Kwak JY, Kim EK, Chung WY, et al. Association of BRAFV600E mutation with poor clinical prognostic factors and US features in Korean patients with papillary thyroid microcarcinoma. Radiology 2009;253:854-60.

13. Millington GW. Mutations of the BRAF gene in human cancer, by Davies et al. (Nature 2002; 417: 949-54). Clin Exp Dermatol 2013;38:222-3.

14. Liu Z, Lv T, Xie C, et al. BRAF V600E Gene Mutation Is Associated With Bilateral Malignancy of Papillary Thyroid Cancer. Am J Med Sci 2018;356:130-4.

15. Kim TH, Park YJ, Lim JA, et al. The association of the $\mathrm{BRAF}(\mathrm{V} 600 \mathrm{E})$ mutation with prognostic factors and poor clinical outcome in papillary thyroid cancer: a metaanalysis. Cancer 2012;118:1764-73.

16. Kebebew E, Weng J, Bauer J, et al. The prevalence and prognostic value of BRAF mutation in thyroid cancer. Ann Surg 2007;246:466-70; discussion 470-1.

17. Luo J, Zhang C, Huang F, et al. Risk of malignancy in thyroid nodules: predictive value of puncture feeling of grittiness in the process of fine-needle aspiration. Sci Rep 2017;7:13109.

18. İnci MF, Özkan F, Yüksel M, et al. The effects of sonographic and demographic features and needle size on obtaining adequate cytological material in sonographyguided fine-needle aspiration biopsy of thyroid nodules. Endocrine 2013;43:424-9.

19. Smith-Bindman R, Lebda P, Feldstein VA, et al. Risk of thyroid cancer based on thyroid ultrasound imaging characteristics: results of a population-based study. JAMA Intern Med 2013;173:1788-96.

20. Asteria C, Giovanardi A, Pizzocaro A, et al. USelastography in the differential diagnosis of benign and malignant thyroid nodules. Thyroid 2008;18:523-31.

21. Yan C, Huang M, Li X, et al. Relationship between BRAF 
V600E and clinical features in papillary thyroid carcinoma. Endocr Connect 2019;8:988-996.

22. Haroon Al Rasheed MR, Xu B. Molecular Alterations in Thyroid Carcinoma. Surg Pathol Clin 2019;12:921-30.

23. Frasca F, Nucera C, Pellegriti G, et al. BRAF(V600E) mutation and the biology of papillary thyroid cancer. Endocr Relat Cancer 2008;15:191-205.

24. Hayashida N, Namba H, Kumagai A, et al. A rapid and simple detection method for the BRAF(T1796A) mutation in fine-needle aspirated thyroid carcinoma cells. Thyroid 2004;14:910-5.

Cite this article as: Li L, Li P, Chen X, Kang L, Ye Y. Diagnostic value of puncture feeling combined with BRAF V600E mutation in repeat US-FNA biopsy of Bethesda III thyroid nodules. Gland Surg 2021;10(6):2019-2027. doi: $10.21037 / g s-21-302$
25. Brown AE, Lim KS, Corpus G, et al. Detection of BRAF mutation in the cytocentrifugation supernatant fluid from fine-needle aspiration of thyroid lesions may enhance the diagnostic yield. Cytojournal 2017;14:4.

26. Su X, Jiang $X, X u X$, et al. Diagnostic value of BRAF (V600E)-mutation analysis in fine-needle aspiration of thyroid nodules: a meta-analysis. Onco Targets Ther 2016;9:2495-509.

(English Language Editor: C. Betlazar-Maseh) 